\title{
LONG-TERM SURGICAL OUTCOMES OF PATIENTS WITH PHACOMORPHIC GLAUCOMA
}

\author{
Mustafa Ömer İzzettinoğlu ${ }^{1}$ (D), Ayça Küpeli Çınar² (D), Rüveyde Garip ${ }^{2}$
}

${ }^{1}$ Trakya University School of Medicine, Edirne, TURKEY

${ }^{2}$ Department of Ophthalmology, Trakya University School of Medicine, Edirne, TURKEY

\begin{abstract}
Aims: To retrospectively analyze clinical characteristics of patients diagnosed with phacomorphic glaucoma in the Ophthalmology Department of Trakya University Hospital. Methods: This retrospective cross-sectional study was conducted with patients who were diagnosed with phacomorphic glaucoma in the Ophthalmology Department of Trakya University Hospital between January 2010 and January 2021. Results: Initially, 19 eyes from 19 patients diagnosed with phacomorphic glaucoma met the inclusion criteria. The mean preoperative best-corrected visual acuity was $0.1 \pm 0.3$ Snellen visual acuity (ranging from 0.001 to 1.0 Snellen visual acuity). A statistically significant increase in postoperative best-corrected visual acuity during the follow-ups has been observed. In terms of intraocular pressure levels, patients had mean preoperative $32.3 \pm 11.2 \mathrm{mmHg}$ (range:15-55 mmHg). The decrease in postoperative intraocular pressure levels during follow-ups was statistically significant. Conclusion: Lens removal through phacoemulsification or intracapsular cataract extraction is an effective and safe procedure in the treatment of phacomorphic glaucoma, ensuring a satisfactory long-term intraocular pressure control and a rapid functional recovery. However, most of the patients are expected to have an outcome of favorable best-corrected visual acuity after surgery in the long term. Keywords: Cataract, phacomorphic glaucoma, epidemiology, glaucoma
\end{abstract}

\section{INTRODUCTION}

Phacomorphic glaucoma (PG) is secondary angle-closure glaucoma that results from mature cataract formation. The narrowing of the angle between the iris and the cornea could occur slowly with cataract formation by a forward push of the iris or acute precipitation by an intumescent cataractous lens which leads to obstruction of aqueous flow between the border of the anterior capsule of the lens and the pupil $(1,2)$. The three main causes of blindness have been reported to be cataract, trachoma, and glaucoma; together accounting for two-thirds (71\%) of all blindness (3). Cataract has been reported to be the single largest cause of bilateral blindness (15.83 million) on a global scale, and the backlog of unoperated cataract cases have exceeded the number that was estimated in 1990 by World Health Organization Consultation (3). PG happens to be more common in developing countries due to the lack of awareness of cataracts as a disease and delayed lens extraction operations (4).

The risk factors for PG were determined to be age being greater than 60 years, shallow anterior chamber, and axial length shorter than $23.7 \mathrm{~mm}$ (5). The patients with PG mostly present to the clinics with pain in the eye, a history of decreased vision, evidence of mature cataract formation, angle-closure, and elevated intraocular pressure (IOP) in the affected eye. The presence of an intumescent cataractous lens and the presence of cell and flare essentially play the biggest role in the distinction between primary angle-closure glaucoma and phacomorphic angle-closure glaucoma (1).

Initial PG therapy includes medical treatment such as topical prostaglandins, carbonic anhydrase inhibitors, beta-blockers, and hyperosmotic agents such as intravenous mannitol or oral glycerin.
An ophthalmology specialist should use parasympathomimetic agents with caution since these agents can cause pupillary blockage. If the medical treatment alone is not enough to control the IOP, laser iridotomy is usually performed and other options include corneal depression with a Zeiss 4-mirror lens. If the eye with no symptoms is also predisposed to angle-closure glaucoma, prophylactic laser iridotomy is suggested to be performed on the fellow eye (1, 2). Cataract extraction is the definitive treatment of PG (1). It is highly important to raise awareness on cataracts and the outcomes of the disease to recognize the patients with PG as early as possible and apply the correct ophthalmological procedures when PG is detected.

This retrospective study aims to analyze the clinical characteristics, examination findings, and outcomes of the patients diagnosed with PG in the tertiary ophthalmology clinic of Trakya University Hospital.

\section{MATERIAL AND METHODS}

This retrospective cross-sectional study was approved by the Scientific Research Ethics Committee of Trakya University School of Medicine (Protocol Code: TÜTF-BAEK 2021/115). The study was carried out in accordance with the tenets of the Declaration of Helsinki. Written informed consent for the use of medical information of patients was also received from all of the participants.

Our study analyzed 19 eyes of 19 patients who were diagnosed with PG and whom the cataract extraction surgery was performed in the Ophthalmology Department of Trakya University Hospital between January 2010 and January 2021. Demographic data such 
as age and gender, duration of symptoms, accompanying systemic comorbidities such as hypertension, diabetes, the usage of topical prostaglandins, beta-blockers, carbonic anhydrase inhibitors, and alpha agonists, and initial and following clinical findings were obtained from the medical records of the patients. All patients underwent complete ophthalmologic examination at each visit including best-corrected visual acuity (BCVA) determined by Snellen chart, anterior segment biomicroscopic examination, IOP measurement with Goldmann applanation tonometer, and detailed fundus examination obtained with 78-diopters non-contact lens.

Individuals diagnosed with PG were included in this study. The ones who have underlying corneal pathologies, ocular-vascular diseases (central retinal artery occlusion, retinal venous occlusion), accompanying macular pathologies like a macular hole and macular degeneration that could lead to unreliable examinational results were excluded from the study.

The following findings were documented at the initial visit: BCVA, IOP, ocular comorbidities such as central retinal vein occlusion, pseudoexfoliation syndrome, and presence of glaucoma.

The following findings were documented at the follow-up visits: performed surgical technique of cataract extraction, the usage and the duration of the usage of topical anti-glaucomatous agents, kinds of anti-glaucomatous agents that have been used, measurements of BCVA and IOP of the postoperative first day, first week, first month, and the third month, and the presence of postoperative complications.

Surgical success was determined when postoperative IOP decrease and postoperative BCVA increase were achieved. In the present study, postoperative IOP less than $20 \mathrm{mmHg}$ and one decimal increase in the Snellen line in terms of BCVA were considered surgical achievements.

\section{Statistical Analysis}

The collected data were analyzed statistically by using IBM SPSS Statistic 20 for Windows (Version 20.0. Armonk, NY: IBM Corp.). Mean and standard deviation values were calculated using descriptive statistical measures. The frequency distribution of qualitative data was quantified in percentages. The Chi-square test was used for qualitative comparison. Normality distribution of the data was evaluated with the Shapiro-Wilk test. Quantitative data were compared with the Student t-test. Friedman test was used to compare repeated measures more than 2 in quantity when normal distribution was rejected. Patients with negative light perception were not included in the statistical analysis. P-value of $<0.05$ was considered to be statistically significant.

\section{RESULTS}

Initially, 19 eyes from 19 patients with the diagnosis of PG met the inclusion criteria. Eleven (57.9\%) patients were female, and 8 (42.1\%) patients were male. The summary of patients' characteristics including mean age, mean duration of symptoms, gender, number of the affected eye and its side, and used topical anti-glaucomatous agents are presented in Table 1.

Five $(26.3 \%)$ patients were under the usage of carbonic anhydrase inhibitor, six (31.6\%) patients received beta-blockers. Eight (42.1\%) patients received phacoemulsification and eleven (57.9\%) patients underwent intracapsular cataract extraction surgery.

The mean preoperative BCVA was $0.1 \pm 0.3$ Snellen visual acuity (ranging from 0.001 to 1.0 Snellen visual acuity). Statistically significant increase in postoperative BCVA during the follow-ups has been observed $(\mathrm{p}<0.001$, Friedman test).

In terms of IOP levels, patients had a mean preoperative IOP of $32.3 \pm 11.2 \mathrm{mmHg}$ (ranging from 15 to $55 \mathrm{mmHg}$ ). The decrease in postoperative IOP levels during follow-ups was statistically significant $(\mathrm{p}<0.001$, Friedman test). All patients had postoperative third-month IOP levels less than $20 \mathrm{mmHg}$.

In terms of postoperative complications, $9(47.4 \%)$ patients had corneal edema, 4 (21.1\%) patients had Descemet folds, 2 (10.5\%) patients had hyphema, $1(5.3 \%)$ patient had pupillary blasted exudation, and $3(15.8 \%)$ patients had no complications at all. Detailed and precise information of all patients in this study conducted with a small number of patients in terms of BCVA and IOP are shown in Table 2 to give a better perspective on the prognosis of the patients.

Table 1: Patient characteristics.

Number of Patients [n (\%)]

\begin{tabular}{lc}
\hline Age $(\text { years })^{*}$ & $78.2 \pm 7.9(53-88)$ \\
Duration of Symptoms (days)* & $64.6 \pm 115.4(1-365)$ \\
Gender & $11(57.9)$ \\
Female & $8(42.1)$ \\
Male & $10(52.6)$ \\
Eye Side & $9(47.4)$ \\
Right & \\
Left & $5(26.3)$ \\
Topical anti-Glaucomatous Agent & $6(31.6)$ \\
CAI & $8(42.1)$ \\
Beta Blocker & \\
None & \\
\hline
\end{tabular}

CAI: Carbonic anhydrase inhibitor

${ }^{*}$ Data were expressed as mean $\pm S D$ (min-max). 
Table 2: Preoperative and postoperative BCVA in Snellen decimal of all patients.

\begin{tabular}{|c|c|c|c|c|c|}
\hline Patient Number & Pre-op & Post-op Day 1 & Post-op Week 1 & Post-op Month 1 & Post-op Month 3 \\
\hline 1 & 0.0010 & 0.0300 & 0.10 & 0.20 & 0.40 \\
\hline 2 & 0.0010 & 0.0010 & 0.0010 & 0.0500 & 0.0500 \\
\hline 3 & 0.0010 & 0.0050 & 0.0300 & 0.10 & 0.20 \\
\hline 4 & 0.60 & 0.20 & 0.70 & 0.0500 & 0.60 \\
\hline 5 & 0 & 0 & 0 & 0 & 0 \\
\hline 6 & 0.0001 & 0.0010 & 0.0010 & 0.0010 & 0.0010 \\
\hline 7 & 0.0160 & 0.0010 & 0.0160 & 0.20 & 0.0010 \\
\hline 8 & 0 & 0 & 0 & 0 & 0 \\
\hline 9 & 1 & 0.0030 & 0.30 & 0.50 & 0.70 \\
\hline 10 & 0.0010 & 0.0010 & 0.30 & 0.40 & 0.70 \\
\hline 11 & 0.0001 & 0.0001 & 0.0001 & 0.0001 & 0 \\
\hline 12 & 0.0001 & 0.0010 & 0.0010 & 0.0300 & 1 \\
\hline 13 & 0.0010 & 0.0010 & 0.0010 & 0.0010 & 0.0010 \\
\hline 14 & 0.0010 & 0.0160 & 0.20 & 0.70 & 0.70 \\
\hline 15 & 0.0001 & 0.0010 & 0.40 & 0.60 & 0.0010 \\
\hline 16 & 0 & 0 & 0 & 0 & 0.70 \\
\hline 17 & 0.0010 & 0.0010 & 0.0080 & 0.10 & 0.0160 \\
\hline 18 & 0.0010 & 0.0010 & 0.0010 & 0.0300 & 0.10 \\
\hline 19 & 0.0001 & 0.0010 & 0.0500 & 0.0500 & 0.0500 \\
\hline Mean \pm Standard Deviation & $0.10 \pm 0.25$ & $0.02 \pm 0.44$ & $0.13 \pm 0.19$ & $0.19 \pm 0.22$ & $0.28 \pm 0.33$ \\
\hline Median & 0.001 & 0.001 & 0.008 & 0.050 & 0.050 \\
\hline Maximum & 1 & 0.20 & 0.70 & 0.70 & 1 \\
\hline Minimum & 0 & 0 & 0 & 0 & 0 \\
\hline
\end{tabular}

BCVA: Best-corrected visual acuity, Pre-op: Preoperative, Post-op: Postoperative

\section{DISCUSSION}

Cataract is known to be the most important cause of blindness in developing countries, mostly occurring in elder rural populations (6). Unoperated cataractous lenses eventually lead to major complications of PG causing poor vision and high IOP. This delay of lens extraction mostly happens due to the lack of awareness about the significance of early cataract management in developing countries (6). PG is secondary angle-closure glaucoma which is resulted from mature cataract formation. With a forward push of the iris or acute precipitations of the intumescent cataractous lens, narrowing of the angle leads to high IOP levels (7).

Phacomorphic glaucoma mostly occurs in elderly patients with delayed lens extraction and rarely in the young population with a genetically large lens (7). In the present detailed study, it was revealed that the range of the age of patients with $\mathrm{PG}$ was at the range of 53-88 years with the mean age of 78.2 years, which was consistent with the findings of other studies $(6,7)$. Regarding gender, female patients are predominant to PG according to the present study. Demographic data of other studies have also shown similar results of females making up the majority of the total group with PG (6-8). The predominance of females to have PG is thought to exist due to the socioeconomic repression and the fact that cataract is more prevalent to occur in female individuals (7). In the present study, it was shown that symptoms of the patients that were presented to the clinic with vision impairment, eye pain, eye redness, or high IOP had lasted averagely for 64.6 days. The study of Kothari et al. (6) has revealed that the duration of the onset PG symptoms in days was mostly 3 days or more than 14 days with two peaks, which are considerably less than the symptom duration of the patients in the present study. Furthermore, according to the results of the retrospective study that was conducted by Pradhan et al. (9), most of the patients had 10 days with onset symptoms of PG, which is again noticeably less than our results. Poor awareness of patients could be held accountable for the longer duration of the symptoms that was shown by the results of the present study.

Patients with PG occur to have severe visual impairments due to mature cataract formation and high IOP. In our study, it was seen that the patients had a mean BCVA of 0.10 decimals. In the study of Pradhan et al. (9), patients have had a preoperative BCVA of 0.32 decimals with subtly having better BCVA results, and, in another study conducted by Prajna et al. (8), patients were observed to have BCVA of 0.10 decimals which is the same result as the present study. As the cataract matures and surgery is delayed, IOP increases by narrowing the anterior chamber angle and causes difficulties in surgical procedures. The frequency of corneal edema increases depending on phacoemulsification power. The rates of inflammation seen in the eyes after ingestion of ripe cataracts are also higher (1). In our study, patients had a worsened BCVA at discharge from surgery (first-day examination) due to the inflammation, but on the contrary postoperative BCVA results happened to have a statistically significant increase. Various studies also showed that postoperative states of BCVA have been considerably better than the presented BCVA results (8-12). 
Enlargement of the lens gives a push to the iris, resulting in the narrowing of the border between pupil margin and anterior capsule. Additionally, acute precipitations of the intumescent cataractous lens also cause angle narrowing. These two incidents lead to high IOP levels which are also called phacomorphic angle-closure or lens-induced glaucoma $(1,2)$. In the present study, it was revealed that the patients had a mean preoperative IOP of 32.3 $\mathrm{mmHg}$. Many studies revealed similar results as the results of the present study $(9,11,12)$. The first choice of treatment for high IOP in PG should be with medication, even though surgical removal of the lens is the definitive treatment. The response of PG to the treatment with medication is positive which results in lower IOP. The first approach to PG with medication treatment provides patients to have stable postoperative IOP without any need for further anti-glaucomatous medications when near normal to a normal level of IOP is achieved. According to our results, postoperative IOP has been observed to drop to the normal range, having an IOP lower than $21 \mathrm{mmHg}$, which was coherent to many other studies (8-12).

Phacomorphic glaucoma should be initially treated with medicinal approaches for a specific period before surgical procedures for a better outcome and sustained postoperative IOP. Nevertheless, the definitive treatment for PG is lens extraction surgery (5). In the present study, patients underwent intracapsular cataract extraction (ICCE) and phacoemulsification cataract extraction. As the results have shown, lens extraction has an important and positive impact on the prognosis of BCVA and IOP. The study of Moraru et al. (5) also pointed out the importance of lens extraction surgery in the prognosis of PG. Corneal edema and anterior chamber inflammation with a fibrous reaction on the first few days are the complications that specialists frequently come across in the early postoperative period. In the present study, corneal edema was observed on $47.4 \%$ of the patients but successfully treated with topical agents. Corneal edema was also shown to frequently occur in the early postoperative period by the study of Moraru et al. (5).

The main three limitations of the present study are the small number participating patients with $\mathrm{PG}$, lack of data of premedication phase IOP due to being a tertiary ophthalmology clinic, and application of different types of lens extraction techniques such as phacoemulsification and ICCE. Missing surgical data of patients who underwent ICCE surgery on whether scleral fixation or anterior vitrectomy is received is another limitation to the present study and it is due to the application of old case files of participating patients. Procedures such as scleral fixation and anterior vitrectomy could also notably affect the outcomes of the patients who underwent these procedures. In addition, it is worth mentioning that being a tertiary ophthalmology clinic, patients come to us with referrals from other departments and are already treated with anti-glaucomatous agents. Thus, it challenges us to get the data of intraocular pressures of patients before medical treatment. Not all patients could be subjected to phacoemulsification due to challenging difficulties leading the specialists to proceed with ICCE or extracapsular cataract extraction. In our study, a certain number of patients were operated with the ICCE technique, which impacts the resulting BCVA due to bigger corneal incisions.
In conclusion, PG is angle-closure glaucoma that occurs in the basement of delayed lens extraction surgery and a mature cataract or intumescent cataractous lens. Therefore, individuals with a lens that has an increased density should undergo lens extraction surgery before significant complications occur. Lens removal through phacoemulsification or ICCE is an effective and safe procedure in the treatment of PG, ensuring satisfactory long-term IOP control and a rapid functional recovery. However, the rate of postoperative complications and the outcomes are better in patients treated with phacoemulsification. Nevertheless, not every case could be indicated to this type of surgical technique due to inoperative difficulties associated with the angle-closure. However, most of the patients are expected to have an outcome of favorable BCVA after surgery in the long term. Further studies are needed to thoroughly reveal the relationship between the treatment regimens and the progression and prognosis of the disease.

Ethics Committee Approval: This retrospective study was approved by the Scientific Research Ethics Committee of Trakya University School of Medicine (Protocol Code: TÜTF-BAEK 2021/115).

Informed Consent: Informed consent was obtained from all of the subjects. Conflict of Interest: The authors declared no conflict of interest.

Author contributions: Concept: MÖİ, AKÇ, RG. Design: MÖİ, AKÇ, RG. Supervision: MÖİ, AKÇ, RG. Resources: MÖİ, AKÇ, RG. Materials: MÖİ, AKÇ, RG. Data collection and/or processing: MÖİ, AKÇ, RG. Analysis and/ or Interpretation: MÖİ, AKÇ, RG. Literature Search: MÖİ, AKÇ, RG. Writing Manuscript: MÖİ, AKÇ, RG. Critical Review: MÖİ, AKÇ, RG.

Financial disclosure: The authors declared that this study received no financial support.

\section{REFERENCES}

1. Ellant JP, Obstbaum SA. Lens-induced glaucoma. Doc Ophthalmol 1992;81(3):317-38.

2. Kanski J, Brad Bowling. Lens-related glaucoma. In: Kanski J, Brad Bowling, editors. Clinical Ophthalmology: A systemic approach. 7th ed. New York: Elsevier/ Saunders; 2011.

3. Thylefors B, Négrel AD, Pararajasegaram R et al. Global data on blindness. Bull World Health Organ 1995;73:115-21.

4. Ramakrishanan R, Maheshwari D, Kader MA et al. Visual prognosis, intraocular pressure control and complications in phacomorphic glaucoma following manual small incision cataract surgery. Indian J Ophthalmol 2010;58(4):303-6.

5. Moraru A, Pînzaru G, Moțoc A et al. Functional results of cataract surgery in the treatment of phacomorphic glaucoma. Rom J Ophthalmol 2017;61(3):202-6.

6. Kothari R, Tathe S, Gogri P et al. Lens-induced glaucoma: the need to spread awareness about early management of cataract among rural population. ISRN Ophthalmol 2013;2013:581727.

7. Potop V, Coviltir V, Schmitzer S et al. Ultrasound biomicroscopy as a vital tool in occult phacomorphic glaucoma. Rom J Ophthalmol 2019;63(4):311-4.

8. Prajna NV, Ramakrishnan R, Krishnadas R et al. Lens induced glaucomas-visual results and risk factors for final visual acuity. Indian J Ophthalmol 1996;44(3):14955.

9. Pradhan D, Hennig A, Kumar J et al. A prospective study of 413 cases of lens-induced glaucoma in Nepal. Indian J Ophthalmol 2001;49(2):103-7.

10. Sharanabasamma M, Vaibhav K. Management and visual outcome in patients of lens-induced glaucomas at a tertiary eye care hospital in South India. J Curr Glaucoma Pract 2016;10(2):68-75.

11. Angra SK, Pradhan R, Garg SP. Cataract induced glaucoma - an insight into management. Indian J Ophthalmol 1991;39(3):97-101.

12. Lee JW, Lai JS, Yick DW et al. Prospective study on retinal nerve fibre layer changes after an acute episode of phacomorphic angle closure. Int Ophthalmol 2012;32(6):577-82. 